\title{
Stress Among Iranian Nurses in Critical Wards
}

\author{
Seyed Mohammad Reza Hashemian ${ }^{1}$; Behrooz Farzanegan ${ }^{2}$; Mohammad Fathi ${ }^{3}$; \\ Seyed Hossein Ardehali ${ }^{4}$; Amir Vahedian-Azimi ${ }^{5}$; Mohammad Asghari-Jafarabadi ${ }^{6}$; \\ Mohammadreza Hajiesmaeili ${ }^{7, *}$ \\ ${ }^{1}$ Chronic Respiratory Diseases Research Center (CRDRC), National Research Institute of Tuberculosis and Lung Diseases (NRITLD), Masih Daneshvari Hospital, Shahid Beheshti \\ University of Medical Sciences, Tehran, IR Iran \\ ${ }^{2}$ Tracheal Diseases Research Center (TDRC), Masih Daneshvari Hospital, Shahid Beheshti University of Medical Sciences, Tehran, IR Iran \\ ${ }^{3}$ Shahid Modares Hospital, Shahid Beheshti University of Medical Sciences, Tehran, IR Iran \\ 4 Anesthesiology Research Center, Shohada Tajrish Hospital, Shahid Beheshti University of Medical Sciences, Tehran, IR Iran \\ 5 Anesthesiology Research Center, Shohada Tajrish Hospital, Shahida Beheshti University of Medical Sciences, Tehran, \\ ${ }_{6}$ Behavioral Sciences Research Center, Nursing Faculty, Baqiyatallah University of Medical Sciences, Tehran, IR Iran
7 Traffic Injury Prevention Research Center, Faculty of Health, Tabriz University of Medical Sciences, Tabriz, IR Iran \\ ${ }^{7}$ Clinical Research Development Center, Loghman Hakim Hospital, Shahid Beheshti University of Medical Sciences, Tehran, IR Iran \\ ${ }^{*}$ Corresponding Author: Mohammadreza Hajiesmaeili, Clinical Research Development Center, Loghman Hakim Hospital, Shahid Beheshti University of Medical Sciences, P.O. \\ Box: 1333635445, Tehran, IR Iran. Tel: +98-2151025343, Fax:+98-2155424040, E-mail: mrhajiesmaeili@sbmu.ac.ir
}

Received: August 7, 2014; Revised: February 8, 2015; Accepted: March 25, 2015

\begin{abstract}
Background: Stress has been recognized as a significant psychosocial and physiologic component in educational and practical processes. Objectives: The purpose of present study was to survey stress among Iranian nurses in critical wards.

Patients and Methods: In this cross-sectional study, 3643 anonymous questionnaires were disseminated among nurses in different hospitals in Tehran, Iran, by utilizing multistage random sampling. The questionnaire consisted of two sections: demographic characteristics and a 22-item list on a five-point Likert scale. The association of variables with stress was evaluated using hierarchical logistic regression.

Results: A total of 3043 completed questionnaires were completed and returned (response rate, 83.53\%). Age ( $>33$ years, 1571 (51.63\%); $<$ 33 years old, 1472 (48.37\%)), marital status (single, 188 (6.18\%); married, 2685 (88.24\%); and widow, 170 (5.59\%)), working shift (morning, 605 (19.88\%); evening, 631 (20.74\%); night, 603 (19.82\%); and rotation, 1204 (39.57\%)), and the years of experience of nurses (1- 5 years, 413 (13.57\%); 6 - 10 years, 589 (19.36\%); 11 - 15 years, 832 (27.34\%); 16 - 20 years, 758 (24.91\%); and 21-25 years, 451(14.82\%)) had significant association with the level of stress ( $\mathrm{P}<0.05$ for all parameters). However, sex (male, 937 (30.79\%); and female, 2106 (69.21\%)), education (associate, 444 (14.59\%); baccalaureate, 2250 (73.94\%); and master, 349 (11.47\%)), and body mass index (>24.6 kg/m² 2514 (49.75\%); and <24.6 kg/m², 1529 (50.25\%)) showed no significant association with the level of stress $(\mathrm{P}>0.05$ for all parameters).

Conclusions: Considering the personal and background characteristics of personnel and understanding their association with stress and stressful situations encompasses the ability to persevere and adapt to contextual stressors.
\end{abstract}

Keywords: Intensive Care Unit; Coronary Care Unit; Nursing; Iran

\section{Background}

Every day we are facing with stress in our private life or public work. Its proper level may help us cope with challenging situations; but the high level of stress may cause anxiety and irritation, and even threat health. Therefore, its consequences can be disappointing.

Nursing is a job with a high level of stress, especially in some wards such as intensive care unit (ICU), coronary care unit (CCU), and dialysis unit $(1,2)$. The crucial responsibilities of ICU and CCU nurses, operating with highly developed technologies, and necessitating fast decision making in ICU patients, excessive workload (3), management styles, managerial skills, professional disagreement, and the emotional cost of caring (4-6) are the remarkable characteristics of the job of ICU nurses. Therefore, their work is making stress mentally and emotionally; if nurses are not able to handle the surrounding circumstances stressors related to the nursing environment, they may become unable to cope and accordingly, quit working (7-9).

In the last few decades, the work of nurses has attracted scientists' interests from organizational and psychologic perspectives $(10,11)$. To understand the stressors and the way of their resolution, several studies have been conducted. However, regarding social and environmental differences in different hospitals and different social and cultural environments, it is important to evaluate stressors by considering organizational characteristics (12-14). Although little is known about the effects of age, years of experience, and education in association with anticipating low level of stress, these features have been recognized to affect coping and stress management behaviors in different nursing backgrounds $(15,16)$. The result of a

Copyright (C) 2015, Iranian Red Crescent Medical Journal. This is an open-access article distributed under the terms of the Creative Commons Attribution-NonCommercial 4.0 International License (http://creativecommons.org/licenses/by-nc/4.0/) which permits copy and redistribute the material just in noncommercial usages, provided the original work is properly cited. 
study on a small sample of acute surgical nurses showed that there was a positive correlation between age, years of experience, and vulnerability to the effects of workplace stress. Older nurses ( $>51$ years) had the lowest levels of stress (17). Some studies indicated that older nurses more effectively adapt to the workplace stress than their younger colleagues do, and perhaps cope more efficiently with the continuous changes in healthcare industry. Other nursing studies across identical backgrounds tend to corroborate this finding; therefore, these findings indicate that there is a positive correlation between the level of education and years of experience and successful control of workplace stressors $(15,16,18)$.

Excessive workplace stress not only can cause exhaustion, job frustration, and fading morals, but also has negative influence on staff (19). It is valuable to evaluate this issue to determine the factors that can affect employees frame of mind (20).

\section{Objectives}

Regarding the importance of the workplace stressors, especially in nursing care, a survey in the different critical units (dialysis unit, CCU, and ICU) of different hospitals in Tehran, the capital of Iran, was done to compare nurses' stressors in different populations and its association with some background and personal variables. This comparison allows further investigation in coping approach, which might maintain nurses' health.

\section{Patients and Methods}

In this cross-sectional study, 3643 anonymous questionnaires were distributed to a sample of nurses in dialysis unit, CCU, and ICU from a list of those who were working in these critical wards of the hospitals of educational and non-educational hospitals in Tehran, Iran. Data were collected from March 2012 to January 2014. The study had three inclusion criteria including working in critical care units, willingness to participate in the study, and availability of the full length completed questionnaire on the referring time of researcher. Finally, 3043 completed and useful questionnaires were returned (response rate, 83.53\%) (Table 1).

The exclusion criterion was unavailability of fully completed questionnaire. The sample size was determined based on information from a pilot study of 100 samples.
An odds ratio (OR) equal to 0.90 for the association between years of experience and stress levels had been found. By considering this value of OR; with a proportion of stress of $0.50,95 \%$ confidence level, and power of $80 \%$, sample size was calculated at 2801 subjects by G-Power 3.1.2 software (available at: www.psycho.nui-duesseldorf. de/abteilungen/aap/gpower3). Considering a dropout rate of $25 \%$, the final sample size was determined to be 3643 subjects. The sampling method was multistage random sampling so that in the first step, 16 hospitals (8 hospitals from educational and 8 hospitals from noneducational referral hospitals) were randomly selected from all hospitals (50 hospitals) and in the second step, a simple random sampling method was used to randomly select the nurses amongst the critical units of hospitals. Sampling framework was received by inquiring about the number of hospitals and working nurses in critical care units according to the predetermined samples. The names of all hospitals in Tehran were collected, divided based on the educational and non-educational ones, and wrote on the identical pieces of paper. Eight hospitals from each educational and same number from noneducational hospitals were selected by simple random sampling with replacement methods until the predetermined number was achieved. In the second step, the list of all working nurses in the critical care units were generated and based on the simple random sampling, the predetermined numbers were selected. The researchers distributed the questionnaires and an information form outlining (enclosed cover letter) the aim of the study among nurses. Confidentiality, voluntary participation, and anonymity were maintained throughout the study. To ensure anonymity, no code numbers were appointed on the questionnaires to identify responders.

The questionnaire consisted of two parts: the first part of the questionnaire was related to demographic characteristics (age, marital status, and sex), work shift, number of years spent in the dialysis unit, CCU, and ICU, body mass index (BMI), and qualifications of the respondents. The second part consisted of a 22-item list outlining previously identified work stressors (Box 1). The respondents were asked to rate the work stressors on a five-point Likert scale ranging from "causes me no stress" to "causes me extreme stress". The minimum and maximum score of the questionnaire were 22 and 110 , respectively.

\begin{tabular}{lcc}
\hline Table 1. Validity and Reliability of the Scale ${ }^{\mathrm{a}}$ & & Reliability \\
\hline Face Validity, Impact Score & $2.09-4.67$ & - \\
Content Validity, CVR & 0.45 & - \\
Content Validity, CVI & 0.83 & - \\
Stability, Test-Retest & - & 0.87 \\
Equivalency, Alternative Form & - & 0.85 \\
Internal Consistency, Cronbach's alpha & - & 0.89
\end{tabular}


Hashemian SMR et al.

Box 1. Stressful Situations Scale with 22 Items

1. Dealing with patients pain and suffering

2. Family presence

3. Heavy workload

4. Relatives reaction

5. Time pressure

6. Communicating bad news

7. The necessity of having continual readiness for emergency procedures

8. Death and dying

9. Staff shortage

10. Non-nursing tasks

11. Patients' reactions

12. Physician not available

13. Instability of patient's clinical condition

14. Lack of resources

15. Working extra hours

16. Physicians' demands

17. Decision making

18. Unpleasant tasks

19. Shift rotation

20. Poor cooperation in dialysis, coronary care unit, and intensive care unit

21. Poor cooperation and communication in other departments

22. Disproportionate between salary and job hardness

The content validity of the questionnaire was ensured through reference to information attained in a review of the literature and peer review by a panel of five psychiatric nurses, ten working nurses in critical care units, five physicians working in ICU, five psychologists, and five administrators for supervision. Information about validity and reliability of the scale are presented in Table 2 . Final version of questionnaire was made by considering comments of 35 person in panel. For determining the cutoff points (having or not having stress), receiver operative characteristic (ROC) analysis was used and based on that, score of 67 was selected. Furthermore, any nurses with 67 or higher score had stress and was labeled as nurse in having stress category and the nurses with stress score less than 67 were categorized in not having stress category.

\subsection{Ethical Issues}

Ethical Committee of Masih Daneshvari Hospital, affiliated with Shahid Beheshti University of Medical Sciences, Tehran, Iran, approved the study by the approval number of SBMU1/REC/1391/90. Moreover, Ethical Committee of each hospital reviewed the study and approved its ethical considerations. We explained the purpose of the study to the nurses. They were completely free to participate, decline participation, or withdraw from the research at any
Table 2. Frequency, Percent and 95\% CI for Study Participant

\begin{tabular}{|c|c|c|c|}
\hline Categories & No. (\%) & $\mathbf{L}$ & $\mathbf{U}$ \\
\hline \multicolumn{4}{|l|}{ Age, $y$} \\
\hline$<33$ & $1571(51.63)$ & 49.85 & 53.40 \\
\hline$>33$ & $1472(48.37)$ & 46.60 & 50.15 \\
\hline \multicolumn{4}{|l|}{ Sex } \\
\hline Male & $937(30.79)$ & 29.15 & 32.43 \\
\hline Female & $2106(69.21)$ & 67.57 & 70.85 \\
\hline \multicolumn{4}{|l|}{ Education } \\
\hline Associate & $444(14.59)$ & 13.34 & 15.85 \\
\hline Baccalaureate & $2250(73.94)$ & 72.38 & 75.50 \\
\hline Master & $349(11.47)$ & 10.34 & 12.60 \\
\hline \multicolumn{4}{|l|}{ Marital Status } \\
\hline Single & $188(6.18)$ & 5.32 & 7.03 \\
\hline Married & $2685(88.24)$ & 87.09 & 89.38 \\
\hline Widow & $170(5.59)$ & 4.77 & 6.40 \\
\hline \multicolumn{4}{|l|}{ Working Shift } \\
\hline Morning & $605(19.88)$ & 18.46 & 21.30 \\
\hline Evening & $631(20.74)$ & 19.29 & 22.18 \\
\hline Night & $603(19.82)$ & 18.40 & 21.23 \\
\hline Rotation & $1204(39.57)$ & 37.83 & 41.30 \\
\hline \multicolumn{4}{|c|}{ Years of Experience, $y$} \\
\hline 1-5 & $413(13.57)$ & 12.35 & 14.79 \\
\hline $6-10$ & $589(19.36)$ & 17.95 & 20.76 \\
\hline $11-15$ & $832(27.34)$ & 25.76 & 28.93 \\
\hline $16-20$ & $758(24.91)$ & 23.37 & 26.45 \\
\hline $21-25$ & $451(14.82)$ & 13.56 & 16.08 \\
\hline \multicolumn{4}{|l|}{ BMI, $\mathrm{kg} / \mathrm{m}^{2}$} \\
\hline$<24.6$ & 1514 (49.75) & 47.98 & 51.53 \\
\hline$>24.6$ & $1529(50.25)$ & 48.47 & 52.02 \\
\hline \multicolumn{4}{|l|}{ Stress score } \\
\hline$<67$ & $1637(53.80)$ & 52.02 & 55.57 \\
\hline$>67$ & $1406(46.20)$ & 44.43 & 47.98 \\
\hline
\end{tabular}

a Abbreviations: L, 95\% CI lower bound; U, 95\% CI upper bound.

time throughout the study. The nurses were assured of the confidentiality of their personal data. Subsequently, they provided the written informed consent form.

\subsection{Statistical Analysis}

All analyses were performed using STATA 10 (Stata Corp, College Station, Texas, the United States) (21). Univariate and multivariate multilevel logistic regression analyses were used to assess the association of predictors including age category, sex, education, marital status, working shift, years of experience, and BMI with the outcome variable of stress level (coded as 1 for scores $>67$ and 0 for scores $<67$ ). The variables were entered in each model by 
making them as indicators due to their qualitative nature. In the univariate analysis, each variable entered in the model individually to assess unadjusted association of the predictor with stress and in the multivariate analysis, all variable were entered simultaneously to evaluate the simultaneous association of the predictors with stress. A compound symmetry covariance structure was used. Parameters were estimated using adaptive GaussHermit quadrature integration procedure. A likelihood ratio (LR) goodness-of-fit test was performed for checking model adequacy. Unadjusted and adjusted OR and their 95\% CIs were presented as the effect sizes of interest. In addition, a trend test was performed for years of experi- ence to evaluate the trend of stress levels as growing the years of experience. P values $<0.05$ were considered as statistically significant.

\section{Results}

\subsection{Description of Study Participants}

Of the total study participants (3043 persons), 1571 persons (51.63\%) were < 33 and 1472 (48.37\%) were $>33$ years old. Participants, consisted of 937 males (30.79\%) and 2106 females (69.21\%). Frequency and the percent of other subjects, for each level of predictors are shown in the Table 3.

Table 3. Results of Unadjusted and Adjusted Multilevel Logistic Regression Analyses for variables related to the Stress ${ }^{\text {a }}$

\begin{tabular}{|c|c|c|c|c|c|c|c|c|}
\hline \multirow[t]{2}{*}{ Categories } & \multicolumn{4}{|c|}{ Unadjusted Results } & \multicolumn{4}{|c|}{ Adjusted Results } \\
\hline & OR & $\mathbf{L}$ & $\mathbf{U}$ & PValue & OR & $\mathbf{L}$ & $\mathbf{U}$ & P Value \\
\hline Age, $y$ & & & & $<0.001$ & & & & 0.718 \\
\hline$<33^{b}$ & 1 & - & - & - & 1 & - & - & - \\
\hline$>33$ & 0.72 & 0.62 & 0.83 & & 1.06 & 0.78 & 1.44 & \\
\hline Sex & & & & 0.383 & & & & 0.019 \\
\hline Male b & 1 & - & - & - & 1 & - & - & - \\
\hline Female & 0.93 & 0.80 & 1.09 & & 0.79 & 0.65 & 0.96 & \\
\hline \multicolumn{9}{|l|}{ Education } \\
\hline Associate $^{\mathrm{b}}$ & 1 & - & - & - & 1 & - & - & - \\
\hline Baccalaureate & 0.84 & 0.68 & 1.02 & 0.084 & 0.91 & 0.71 & 1.16 & 0.452 \\
\hline Master & 0.78 & 0.59 & 1.04 & 0.089 & 0.84 & 0.62 & 1.15 & 0.282 \\
\hline \multicolumn{9}{|l|}{ Marital Status } \\
\hline Single ${ }^{b}$ & 1 & - & - & - & 1 & - & - & - \\
\hline Married & 2.53 & 1.82 & 3.53 & $<0.001$ & 2.51 & 1.79 & 3.52 & $<0.001$ \\
\hline Widowed & 1.98 & 1.27 & 3.09 & 0.003 & 1.96 & 1.23 & 3.12 & 0.005 \\
\hline \multicolumn{9}{|l|}{ Shift } \\
\hline Morning ${ }^{b}$ & 1 & - & - & - & 1 & - & - & - \\
\hline Evening & 0.99 & 0.80 & 1.24 & 0.957 & 0.81 & 0.64 & 1.03 & 0.087 \\
\hline Night & 0.87 & 0.69 & 1.09 & 0.227 & 0.68 & 0.53 & 0.87 & 0.002 \\
\hline Rotation & 0.74 & 0.61 & 0.90 & 0.002 & 0.68 & 0.55 & 0.84 & $<0.001$ \\
\hline \multicolumn{9}{|c|}{ Years of Experience, $y$} \\
\hline Trend $^{\mathrm{c}}$ & 0.85 & 0.80 & 0.90 & $<0.001$ & 0.81 & 0.73 & 0.90 & $<0.001$ \\
\hline $1-5^{b}$ & 1 & - & - & - & 1 & - & - & - \\
\hline $6-10$ & 0.75 & 0.58 & 0.96 & 0.025 & 0.67 & 0.52 & 0.87 & 0.002 \\
\hline $11-15$ & 0.57 & 0.45 & 0.73 & $<0.001$ & 0.46 & 0.35 & 0.61 & $<0.001$ \\
\hline $16-20$ & 0.46 & 0.36 & 0.59 & $<0.001$ & 0.42 & 0.28 & 0.62 & $<0.001$ \\
\hline $21-25$ & 0.58 & 0.45 & 0.76 & $<0.001$ & 0.50 & 0.32 & 0.76 & 0.001 \\
\hline \multicolumn{9}{|l|}{ BMI, kg/m² } \\
\hline$<24.9^{b}$ & 1 & - & - & - & 1 & - & - & - \\
\hline 24.9 & 0.94 & 0.81 & 1.08 & 0.362 & 0.93 & 0.81 & 1.08 & 0.352 \\
\hline
\end{tabular}

\footnotetext{
a Abbreviations: L, 95\% CI lower bound for OR; OR, odds ratio; U, 95\% CI upper bound for OR.

b Reference Category.

C The trend test for years of experience showed a significant decreasing trend for stress levels with higher values of this variable in both unadjusted and adjusted analyses.
} 


\subsection{Univariate and Multivariate Multilevel Logis- tic Regressions}

The results of LR Goodness-of-fit test confirmed the model adequacy (Log likelihood $=-046.8221$, Chi Squared $=0.001$, and $\mathrm{P}>0.05$ ). The covariance structure of compound symmetry consisted of two components including standard deviation (value and standard error [SE) of 0.426 and 0.037 , respectively) and Correlation (value and SE of 0.253 and 0.392 , respectively). The model was adjusted for design effect based on these components.

\subsection{Univariate Multilevel Logistic Regression Analyses}

The results showed that age, marital status, working shift, and the years of experience of nurses had significant association with the level of stress $(\mathrm{P}<0.05$ for all). However, sex, education, and BMI showed no significant association with the level of stress ( $P>0.05$ for all). Based on these results, older nurses ( $>33$ years old) had $28 \%$ (OR $=0.72$; 95\% CI, 0.62-0.83) lower stress than younger nurses. Married and widowed nurses had respectively about 2.5 times ( $\mathrm{OR}=2.53$; 95\% CI, 1.82-3.53) and two times (OR $=1.98$; $95 \%$ CI, 1.27-3.09) higher stress than single nurses. Nurses with rotation shift had lower levels of stress ( $O R=$ $0.74 ; 95 \%$ CI, 0.61-0.90). In addition, nurses with longer experience showed lower stress level than nurses with one to five years of experience did, so that nurses with six to ten years $(\mathrm{OR}=0.75 ; 95 \% \mathrm{CI}, 0.58-0.96), 11$ to 15 years $(\mathrm{OR}=$ $0.57 ; 95 \% \mathrm{CI}, 0.45-0.73), 16$ to 20 years $(\mathrm{OR}=0.46$; $95 \% \mathrm{CI}$, $0.36-0.59)$, and 21 to 25 years $(\mathrm{OR}=0.58 ; 95 \% \mathrm{CI}, 0.45-0.76)$ had lower levels of stress than those with one to five years of experience did. In addition, the results of trend test for higher values of this variable confirmed a decreasing trend for stress levels ( $\mathrm{OR}=0.85 ; 95 \% \mathrm{CI}, 0.80-0.90)$.

\subsection{Multivariate Multilevel Logistic Regression Analyses}

In this analysis, all variables were entered in the model to evaluate the simultaneous association of predictors with stress. The results showed that age was not a significant contributor in this analysis $(\mathrm{P}>0.05)$ although it was significant in univariate analysis. In addition, sex of nurses showed a significant association with stress in this analysis $(\mathrm{P}<0.05)$ although it was not significant in univariate analysis. Based on these results, female nurses had $21 \%$ (OR $=0.79 ; 95 \% \mathrm{CI}, 0.65-0.96)$ lower stress than male nurses. Married and widowed nurses had respectively 2.5 times $(\mathrm{OR}=2.51 ; 95 \% \mathrm{CI}, 1.79-3.52)$ and two times $(\mathrm{OR}=1.96$; 95\% CI, 1.23-3.12) higher stress than single nurses. Nurses with night and rotation shift were in lower levels of stress $(\mathrm{OR}=0.68$; 95\% CI, 0.53-0.87; and OR = 0.68; 95\% CI, 0.550.84 , respectively). In addition, nurses with higher experience showed lower stress level than the nurses with one to five years of experience. Nurses with six to ten years $(\mathrm{OR}=0.67 ; 95 \% \mathrm{CI}, 0.52-0.87), 11$ to 15 years $(\mathrm{OR}=0.46 ; 95 \%$
CI, 0.35-0.61), 16 to 20 (OR = 0.42; 95\% CI, 0.28-0.62), and 21 to 25 years $(\mathrm{OR}=0.50 ; 95 \% \mathrm{CI}, 0.32-0.76)$ had lower levels of stress than the nurses with one to five years of experience did. In addition, the results of trend test for higher values of this variable confirmed a decreasing trend for stress levels ( $\mathrm{OR}=0.81 ; 95 \% \mathrm{CI}, 0.73-0.90)$.

\section{Discussion}

Although this research was based on a good sample limited to the hospitals of educational and non-educational in Tehran, Iran, the findings seem to express that many nurses throughout the world have experienced stress in critical units, which is expressed throughout the literature. The Strengths of this research were the used multistage random sampling methods and large sample size. In this research, dialysis ward, CCU, and ICU were considered critical units.

The age of the nurses was identified as a significant factor in explaining stress among nurses working in critical units, which was confirmed by other studies (22-24). The results of this study revealed that older nurses $(>33$ years old) had $28 \%$ lower stress than younger ones ( $<33$ years old). The results of the study also showed that younger nurses experienced more emotional exhaustion and depersonalization (as kinds of stress) than their older colleagues did. While in older nurses personal accomplishment was low and the principle cause of stress, which may be described to nurses later in their career wanting to attain new matters (25). However, other studies contradict this statement because they discovered that personal accomplishment was higher in older nurses and that they know how to supervise and control occupational stress and tension successfully (15). Moreover, research proposes that as age advances, there is a concurrent increase in nurses' capability to handle occupational and workplace stresses across various nursing contexts (15, 26-29).

The results of this study showed that female nurses had $21 \%$ lower stress than male nurses did. However, in a study it was reported that there is no correlation between sex with stress and burnout (chronic stress) (15). Finding of another study showed that female nurses reported higher levels of stress than male nurses did (2). This finding is opposed to our results that may be due to a small sample of their study and limiting samples to one of the educational hospitals of Shahid Beheshti University of Medical Sciences in Iran.

The results of this study indicated that married and widow nurses had respectively 2.5 and two times higher stress than single nurses did. However, in a study no association between age, marital status, and experience variables with decrease or increase of stress was reported (2). This finding contradicts with the results of other studies. One possible reason can explain that single nurses have not extra responsibilities for their home management and have no accountabilities for spouse and children.

The rotation of shift (morning, evening, night, and combinations of them) and nurses' extra working hours were 
recognized as the most significant variables in assessing stress among nurses working in ICU (30), which means working in rotation and night shifts has several adverse effects such as physiologic and psychologic disorders (31$34)$. However, this study did not find that rotation and night shifts were directly related to or directly predicted high levels of stress in nurses working in critical care units. However, it revealed that there was a significant association between low levels of stress and the rotation of shift and night-shift work. However, a considerable proportion of the nurses working in critical care units had more than five years of experience, which may partially explain the different results of this study with other studies. In addition, fatigue due to working was less in fixed shift than in rotation shift. This may be associated with biologic and circadian rhythms coordination with new conditions and circumstances (almost after seven days) (35). The findings of a study showed that working shift times significantly influenced the nine subscale of mental health (somatization, obsessive-compulsive, interpersonal sensitivity, depression, anxiety, aggression, phobia and paranoid). Even after removing the effects of age and years of experience variables from the results, the staff with a fixed shift had a better level of mental health than the staff with a rotation shifts did $(30,36)$. In a study that performed in Iran, the results showed that there was no significant correlation between stress scores and age, work shift, and qualifications (2).

The years of experience of the nurses was recognized as an important variable in assessing stress among nurses working in ICU $(22,23)$. The finding of this research showed a significant association between years of experience and stress levels. In addition, the trend test indicated a significant decreasing trend for stress levels with increasing years of experience in both unadjusted and adjusted analyses. In a study, it was reported that years of experience was the only personal characteristic predictor of adjustment with stress in ICUs (27). Other researches propose that as experience increases, there is a concurrent increase in nurses' capability to handle occupational and workplace stresses across various nursing contexts (15, 26-29).

The strong points of the study were sampling framework, high sample of working nurses in dialysis units, CCU, and ICU, and using advanced statistical methods. It is important to note several limitations of the present study and directions for further research. First, this research was limited by its cross-sectional design. Future research should review the association of other outcome variables such as job stressors with socio-demographic variables over time (for example in a cohort study) in order to address issues of causal relationship. Second, our study depended exclusively on self-report measures. Finally, future research should include the association of stress with other variables such as self-esteem, emotional competence, and coping.

Considering the personnel background, personal char- acteristics and understanding its association with stress and stressful situations encompasses the ability to persevere and adapt to contextual stressors. The results of univariate and multivariate analyses confirmed the marital status, work shift, and especially years of experience as independent factors of stress. In addition, older nurses showed lower stress in univariate analysis and in the multivariate analysis lower stress levels was observed in female nurses.

\section{Acknowledgements}

The authors sincerely thank all the nurses who participated in this study and also, thank to Clinical Research Development Center of Loghman Hakim for methodological helping and support.

\section{Authors' Contributions}

Seyed Mohammad Reza Hashemian, Behrooz Farzanegan, Mohammad Fathi, Seyed Hossein Ardehali, Amir Vahedian-Azimi, Mohammad Asghari Jafarabadi, and Mohammadreza Hajiesmaeili were responsible for the study conception and design, data collection and analysis, and preparing draft of manuscript, and made critical revisions to the paper for important intellectual content and English language editing.

\section{Funding/Support}

This research received full grant from Masih Daneshvari Hospital, Shahid Beheshti University of Medical Sciences, Tehran, Iran (No. SBMU1/REC/1391/90).

\section{References}

1. Beaulieu-Boire G, Bourque S, Chagnon F, Chouinard L, GalloPayet N, Lesur O. Music and biological stress dampening in mechanically-ventilated patients at the intensive care unit ward-a prospective interventional randomized crossover trial. J Crit Care. 2013;28(4):442-50.

2. Adeb-Saeedi J. Stress amongst emergency nurses. Australian Emerg Nurs J. 2002;5(2):19-24.

3. Burnard P, Edwards D, Bennett K, Thaibah H, Tothova V, Baldac chino D, et al. A comparative, longitudinal study of stress in student nurses in five countries: Albania, Brunei, the Czech Republic, Malta and Wales. Nurse Educ Today. 2008;28(2):134-45.

4. Lewis R, Yarker J, Donaldson-Feilder E, Flaxman P, Munir F. Using a competency-based approach to identify the management behaviours required to manage workplace stress in nursing: a critical incident study. Int J Nurs Stud. 2010;47(3):307-13.

5. Littlejohn P. The missing link: using emotional intelligence to reduce workplace stress and workplace violence in our nursing and other health care professions. J Prof Nurs. 2012;28(6):360-8.

6. McCaughey D, DelliFraine JL, McGhan G, Bruning NS. The negative effects of workplace injury and illness on workplace safety climate perceptions and health care worker outcomes. Safety Sci. 2013;51(1):138-47.

7. El-Jardali F, Alameddine M, Dumit N, Dimassi H, Jamal D, Maalouf S. Nurses' work environment and intent to leave in Lebanese hospitals: implications for policy and practice. Int J Nurs Stud. 2011;48(2):204-14.

8. El-Jardali F, Murray SF, Dimassi H, Jamal D, Abualrub R, Al-Surimi $\mathrm{K}$, et al. Intention to stay of nurses in current posts in difficult-tostaff areas of Yemen, Jordan, Lebanon and Qatar: a cross-section- 
al study. Int J Nurs Stud. 2013;50(11):1481-94

9. Wu TY, Fox DP, Stokes C, Adam C. Work-related stress and intention to quit in newly graduated nurses. Nurse Educ Today. 2012;32(6):669-74.

10. Chmiel N. An introduction to work and organizational psychology: a European perspective. John Wiley \& Sons; 2008.

11. Hockey GRJ. Work environments and performance. 2000.

12. Happell B, Reid-Searl K, Dwyer T, Caperchione CM, Gaskin CJ, Burke KJ. How nurses cope with occupational stress outside their workplaces. Collegian. 2013;20(3):195-9.

13. Rickard G, Lenthall S, Dollard M, Opie T, Knight S, Dunn S, et al. Organisational intervention to reduce occupational stress and turnover in hospital nurses in the Northern Territory, Australia. Collegian. 2012;19(4):211-21.

14. Pisaniello SL, Winefield HR, Delfabbro PH. The influence of emotional labour and emotional work on the occupational health and wellbeing of South Australian hospital nurses. J Vocat Behav. 2012;80(3):579-91.

15. Adali E, Priami M. Burnout among nurses in intensive care units, internal medicine wards, and emergency departments in Greek hospitals. ICUs Nurs Web J. 2002;11:1-19.

16. Wong D, Leung S, So C, Lam D. Mental health of Chinese nurses in Hong Kong: The roles of nursing stresses and coping strategies. Online J Issues Nurs. 2001;5(2):1-22.

17. Gillespie BM, Wallis M, Chaboyer W. Operating theater culture: implications for nurse retention. West J Nurs Res. 2008;30(2):259-77.

18. Xianyu Y, Lambert VA. Investigation of the relationships among workplace stressors, ways of coping, and the mental health of Chinese head nurses. Nurs Health Sci. 2006;8(3):147-55.

19. Kang MG, Koh SB, Cha BS, Park JK, Baik SK, Chang SJ. Job stress and cardiovascular risk factors in male workers. Prev Med. 2005;40(5):583-8.

20. Tummers GE, Janssen PP, Landeweerd A, Houkes I. A comparative study of work characteristics and reactions between general and mental health nurses: a multi-sample analysis. J Adv Nurs. 2001;36(1):151-62.

21. Brown H, Prescott R. Applied mixed models in medicine. John Wiley \& Sons; 2006

22. Potter C. To what extent do nurses and physicians working with- in the emergency department experience burnout: A review of the literature. Australas Emerg Nurs J. 2006;9(2):57-64.

23. Koivula M, Paunonen M, Laippala P. Burnout among nursing staff in two Finnish hospitals. J Nurs Manag. 2000;8(3):149-58.

24. Chiriboga DA, Bailey J. Stress and burnout among critical care and medical surgical nurses: a comparative study. CCQ. 1986;9(3):84-92.

25. Gillespie M, Melby V. Burnout among nursing staff in accident and emergency and acute medicine: a comparative study. J Clin Nurs. 2003;12(6):842-51.

26. Moore S, Kuhrik M, Kuhrik N, Katz B. Coping with downsizing: stress, self-esteem and social intimacy. Nurs Manage. 1996;27(3):28.

27. Gillespie BM, Chaboyer W, Wallis M. The influence of personal characteristics on the resilience of operating room nurses: a predictor study. Int J Nurs Stud. 2009;46(7):968-76.

28. Chan E-Y, Morrison P. Factors influencing the retention and turnover intentions of registered nurses in a Singapore hospital. Nurs Health Sci. 2000;2(2):113-21.

29. Tourangeau AE, Cranley LA. Nurse intention to remain employed: understanding and strengthening determinants. J Adv Nurs. 2006;55(4):497-509.

30. Weng BK. Differential relationship of work stress to mental illhealth and job dissatisfaction among nursing professionals. Chin J Psychol. 1991;33:77-86.

31. Williamson AM, Sanderson JW. Changing the speed of shift rotation: a field study. Ergonomics. 1986;29(9):1085-95.

32. Colligan MJ, Smith MJ, Hurrell JJ, Tasto DL. Shiftwork: A record study approach. Behav Res Method Instrum. 1979;11(1):5-8.

33. Parkes D. Sleep Disorders and Neurological Disease. BMJ Publishing Group Ltd; 2000.

34. Jason LA, Wagner L, Rosenthal S, Goodlatte J, Lipkin D, Papernik $\mathrm{M}$, et al. Estimating the prevalence of chronic fatigue syndrome among nurses. Am J Med.1998;105(3A):91S-3S.

35. Jamal M, Jamal SM. Work and nonwork experiences of employees on fixed and rotating shifts: An empirical assessment.J Vocat Behav. 1982;20(3):282-93.

36. Harri M. Mental wellbeing of nurse educators at work. Scand J Caring Sci. 1993;7(2):73-7. 